Gerenciamento de Resultados por Decisões Operacionais no Novo Mercado do Brasil: Uma Análise da Influência de Auditorias Big Four e não Big Four

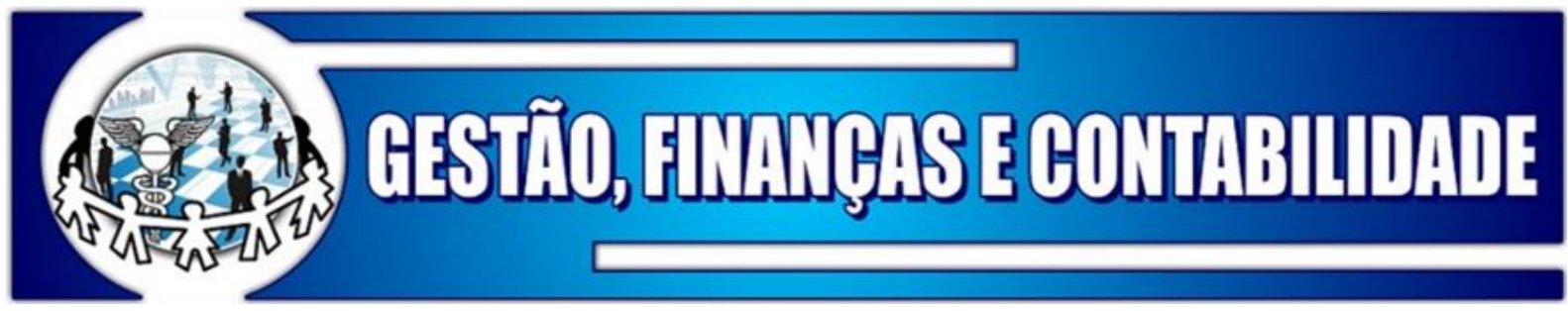

\title{
GERENCIAMENTO DE RESULTADOS POR DECISÕES OPERACIONAIS NO NOVO MERCADO DO BRASIL: UMA ANÁLISE DA INFLUÊNCIA DE AUDITORIAS BIG FOUR E NÃO BIG FOUR
}

\author{
EARNINGS MANAGEMENT BY OPERATIONAL DECISIONS IN THE \\ BRAZILIAN NEW MARKET: AN ANALYSIS OF THE INFLUENCE \\ OF BIG FOUR AND NOT BIG FOUR AUDITS
}

\section{GESTIÓN DE RESULTADOS POR DECISIONES OPERATIVAS EN EL NUEVO MERCADO DE BRASIL: UN ANÁLISIS DE LA INFLUENCIA DE LAS AUDITORÍAS DE BIG FOUR Y NO-BIG FOUR}

Thiago Rios Sena https://orcid.org/0000-0003-0261-7381 Professor na Universidade Federal do Recôncavo da Bahia (UFRB) Mestre em Contabilidade (FCC/UFBA)

E-mail: thiagoriossena@gmail.com

José Maria Dias Filho https://orcid.org/0000-0003-3411-8181 Professor do Programa de Pós-Graduação em Contabilidade (FCC/UFBA) Doutor em Contabilidade e Controladoria (FEA/USP) E-mail: zemariadias@uol.com.br

Nayara Batista Moreira https://orcid.org/0000-0002-0713-7267 Professora na Faculdade Anísio Teixeira (FAT) e Universidade do Estado da Bahia (UNEB) Mestra em Contabilidade (FCC/UFBA) E-mail: nmoreira1105@yahoo.com.br

\section{RESUMO}

Este estudo objetiva verificar a existência de diferenças no nível de gerenciamento de resultados por decisões operacionais nas companhias abertas brasileiras quando auditadas por Big Four ou não Big Four. A amostra de dados apresenta 515 observações para o período de 2012 a 2017, sendo 465 empresas-ano auditadas por Big Four e 50 por não Big Four. A literatura atual aponta a auditoria como um fator inibidor para o gerenciamento de resultado, sendo assim, as hipóteses de pesquisa foram elaboradas a partir desse pressuposto, mas atribuído para o gerenciamento por decisões operacionais. Foi utilizado o modelo proposto por Roychowdhury (2006) para identificar o gerenciamento de resultado através do nível anormal de fluxo de caixa operacional, despesas com vendas, gerais e administrativas e de produção. Os modelos foram operacionalizados através do método dos mínimos quadrados generalizados e os resultados encontrados revelam que o fato da firma de auditoria pertencer ao grupo Big Four não implica 
Sena, Dias Filho e Moreira (2020)

Gerenciamento de Resultados por Decisões Operacionais no Novo Mercado do Brasil:

Uma Análise da Influência de Auditorias Big Four e não Big Four

em diferença no nível de gerenciamento de resultados por decisões operacionais, seja através de fluxo de caixa operacional, despesas de venda, gerais e administrativas ou produção.

Palavras-chave: Gerenciamento de Resultados. Decisões Operacionais. Auditoria. Novo Mercado.

\begin{abstract}
This study aims to verify the existence of differences in the level of earnings management by operational decisions in Brazilian public companies belonging to the São Paulo Stock Exchange when audited by Big Four or not Big Four. The data sample containing 515 observations for the period from 2012 to 2017, being 465 companies-year audited by Big Four and 50 by no Big Four. Current research claims audit can be considered as an inhibiting factor for the management of results, so, the research hypotheses were elaborated from this assumption, but attributed to the management by operational decisions. The model proposed by Roychowdhury (2006) was used to identify the management of results through the abnormal level of operating cash flow, selling, general and administrative and production expenses. The models were operationalized through the generalized least squares method and the results show that the fact that the audit firm belongs to the Big Four group does not imply a difference in the level of earnings management by operational decisions, either through operating cash flow, selling, general and administrative expenses or production.
\end{abstract}

Key words: Earnings Management. Operational Decisions. Audit. New Market.

\title{
RESUMEN
}

Este estudio tiene como objetivo verificar la existencia de diferencias en el nivel de gestión de resultados por decisiones operativas en empresas públicas brasileñas cuando son auditadas por Big Four o no Big Four. La muestra de datos presenta 515 observaciones para el período de 2012 a 2017, siendo 465 empresas-año auditadas por Big Four y 50 por no Big Four. La literatura actual apunta a la auditoría como un factor inhibidor para la gestión de resultados, por lo tanto, las hipótesis de investigación se elaboraron a partir de esta suposición, pero se atribuyeron a la gestión por decisiones operativas. El modelo propuesto por Roychowdhury (2006) se utilizó para identificar la gestión de resultados a través del nivel anormal de flujo de caja operativo, ventas, gastos generales y administrativos y producción. Los modelos se operacionalizaron utilizando el método de mínimos cuadrados generalizados y los resultados muestran que el hecho de que la firma de auditoría pertenezca al grupo Big Four no implica una diferencia en el nivel de gestión de resultados por decisiones operativas, ya sea a través del flujo de caja operativo, gastos de venta, generales y administrativos o producción.

Palabras clave: Gestión de ingresos. Decisiones operacionales. Auditoria. Nuevo mercado.

\section{INTRODUÇÃO}

Um dos principais objetivos da contabilidade é o fornecimento de informações úteis para o processo de tomada de decisões dos diversos agentes econômicos. Esses agentes representam usuários das informações contábeis que encontram nos sistemas de contabilidade mecanismos adequados para subsidiar ou otimizar seus respectivos processos decisórios. (IUDÍCIBUS, 2005; SANTANA et al, 2014).

Partindo dessa premissa, pode-se afirmar que a informação contábil gera implicações econômicas para os mais variados agentes. Peleias (2017) explica que a divulgação da informação contábil é um elemento que auxilia a diminuição do nível da assimetria informacional e aumenta a confiança do usuário em função da transparência.

Para Dechow, Ge e Shrand (2010), a qualidade das informações contábeis está associada com o nível de gerenciamento de resultado da empresa, pois, de acordo com a perspectiva 
Sena, Dias Filho e Moreira (2020)

Gerenciamento de Resultados por Decisões Operacionais no Novo Mercado do Brasil:

Uma Análise da Influência de Auditorias Big Four e não Big Four

oportunística da Teoria Positiva da Contabilidade, os administradores escolhem políticas contábeis para maximizar suas próprias utilidades esperadas referentes a determinada relação de remuneração, contratos de dívidas e custos políticos (WATTS; ZIMMERMAN, 1990). Nesse sentido, o maior nível de gerenciamento de resultado é interpretado como menor qualidade das informações contábeis presentes nos relatórios (DECHOW; SLOAN; SWEENEY, 1996; HEALY; WAHLEN, 1999).

Para Martinez (2013), o gerenciamento de resultados pode se manifestar de diferentes maneiras através da discricionariedade gerencial, entre elas: escolhas contábeis, tomada de decisões operacionais e critérios de apresentação das demonstrações. Diante do exposto, tornase importante garantir a qualidade da informação que é disponibilizada, de modo que ela represente a realidade das transações econômicas ou dos negócios como um todo.

Com base na teoria dos contratos e da agência, os gestores buscam o uso da contabilidade para redução de custos dos contratos a fim de reduzir os custos de agência. Segundo Watts e Zimmerman (1986), no entanto, estes contratos são de pouca utilidade na redução dos custos a menos que a empresa disponha de formas para monitorá-las e executá-las. Auditoria é uma das formas para que os contratos sejam vigiados. Os auditores independentes atuam fundamentalmente como intermediários informacionais, exercendo influência no fluxo de informações para mitigar os custos de agência e reduzir a assimetria informacional (HEALY; PAPELU, 2001).

Braunbeck (2010) estabelece hipótese que relaciona a qualidade das informações contábeis com a qualidade da auditoria, obtendo evidências de que as firmas de auditoria chamadas de "Big-N" oferecem serviços de maior qualidade. Nesse sentido, autores no Brasil como Almeida e Almeida (2009) relatam que as empresas auditadas por Big Four têm menor propensão ao gerenciamento de resultados contábeis, enquanto Martinez (2011) indica que a presença de firmas de autoria Big Four reduz apenas o nível de gerenciamento por decisões contábeis, sem impacto quando analisado o gerenciamento por decisões operacionais para o período de 1998 a 2004.

De acordo com o survey de literatura apresentado por Martinez (2013), a pesquisa envolvendo gerenciamento de resultados nas empresas brasileiras está concentrada naquele referente às escolhas contábeis, operacionalizado por meio dos accruals, sendo incipiente a pesquisa que trata das decisões operacionais como forma de gerenciamento de resultado (MARTINEZ; CARDOSO, 2009; SANTOS; VERHAGEM; BEZERRA, 2011; CUPERTINO, 2013; REIS; CUNHA; RIBEIRO, 2014). Cupertino (2013) afirma que existe um conjunto robusto de evidências quando se trata de empresas que manipulam seus lucros para atingir metas de resultado. Entretanto, a maior parte destas vincula o gerenciamento de resultados aos accruals discricionários, deixando de abordar outras formas de manipulação (XU, 2008; GE, 2010).

Sendo assim, este trabalho busca responder ao seguinte problema de pesquisa: Empresas brasileira de capital aberto auditadas por Big Four possuem menor nível de gerenciamento de resultado por decisões operacionais? Este estudo tem como objetivo verificar se há diferença no nível de gerenciamento de resultados por decisões operacionais nas companhias abertas brasileiras do novo mercado quando auditadas por Big Four ou não Big Four. Para atendimento desse objetivo faz-se necessário discorrer sobre a literatura existente acerca do gerenciamento de resultados, suas formas e fatores inibidores, assim como o papel da auditoria na qualidade das informações contábeis.

Foi identificada uma lacuna acerca da relação da Auditoria Independente como fator inibidor do gerenciamento de resultados por meio de decisões operacionais em empresas brasileiras de capital aberto. Para isso, observou-se o período posterior à adoção da IFRS utilizando o modelo econométrico proposto por Roychowdhury (2006) para identificar o 
Gerenciamento de Resultados por Decisões Operacionais no Novo Mercado do Brasil: Uma Análise da Influência de Auditorias Big Four e não Big Four

gerenciamento de resultado através do nível anormal de fluxo de caixa operacional, despesas com vendas, gerais e administrativas e de produção.

Esse estudo é motivado pela necessidade de evidências empíricas que relacionem outros fatores ao gerenciamento de resultados por decisões operacionais no cenário de mercado emergente. Machado (2018, p. 72) reforça a necessidade de estudos específicos em mercados emergentes, uma vez que "teorias corroboradas por pesquisas em países desenvolvidos podem ter aplicabilidade limitada aos mercados emergentes".

A informação contábil, ao influenciar processos decisórios de diversos grupos, gera importantes implicações de natureza econômica e se faz necessário conhecer os fatores que influenciam no gerenciamento de resultados. Dessa forma, este trabalho busca contribuir para acréscimo à ciência contábil, tanto no tocante ao gerenciamento de resultados por decisões operacionais, como para a literatura acerca da auditoria contábil.

Nas próximas sessões, serão apresentadas: a Revisão de Literatura, abordando os conceitos teóricos que dão suporte à pesquisa, assim como os resultados de outras pesquisas na área; a Metodologia, relatando os procedimentos de coleta, análise e tratamento dos dados; a Análise dos Resultados, discutindo sobre os resultados encontrados na amostra para o cenário brasileiro; e a Conclusão, inserindo as considerações finais da pesquisa.

\section{FUNDAMENTAÇÃO TEÓRICA}

\subsection{GERENCIAMENTO DE RESULTADO}

É possível encontrar na literatura diversos conceitos para Gerenciamento de Resultado, inclusive alguns sinônimos como: Gerenciamento de Lucros, Manipulação (de dados contábeis), Contabilidade Criativa, Gerenciamento de Resultados, "Pedaladas Fiscais", Earning Management, Escolhas Contábeis e Contabilidade Agressiva (MATSUMOTO; PARREIRA, 2007).

O gerenciamento de resultado é uma intervenção proposital no processo de elaboração das demonstrações financeiras externas, com a intenção de obter algum benefício particular, sendo assim oposto ao processo neutro de reportar os resultados contábeis (SHIPPER, 1989). Mais recentemente, Martinez (2013) afirma que o Gerenciamento de Resultados vai além da divulgação das informações contábeis, sendo o uso da discricionariedade gerencial na realização de escolhas contábeis (reconhecimento e mensuração), na tomada de decisões operacionais e na seleção dos critérios de apresentação da demonstração de resultado (disclosure). Ademais, o autor ressalta que tais escolhas devem estar contidas dentro dos limites das normas contábeis, visando influenciar as percepções sobre fatos econômicos subjacentes.

Nota-se que esta última definição é mais extensa que as anteriores, pois contempla outras opções de gerenciamento além daquela fundamentada em escolhas contábeis, bem como não atribui conotação negativa à prática de Gerenciamento de Resultados. Algo importante que é necessário elucidar acerca do gerenciamento de resultados é que ele não constitui fraude contábil. Ao gerenciar resultados, opera-se dentro dos limites da legislação aplicada à contabilidade. Os gestores das empresas exploram os pontos onde as normas contábeis lhes conferem certo grau de discricionariedade, realizando escolhas que não são motivadas apenas pelo interesse de evidenciar a realidade do negócio, mas também em razão de outros incentivos.

Martinez (2001) destaca três tipos de gerenciamento de resultado, cada um deles com objetivos distintos conforme listados a seguir: 1) Target Earnings: gerenciamento de resultados para aumentar ou diminuir os lucros de modo a atingir determinadas metas de referência que podem ser diferentes (maiores ou menores) do resultado do período; 2) Income Smoothing: gerenciamento para diminuir a variabilidade dos resultados e mantê-los em um certo patamar e evitar a sua flutuação; 3) Big Bath Accounting: gerenciar para reduzir os lucros correntes em 
Sena, Dias Filho e Moreira (2020)

Gerenciamento de Resultados por Decisões Operacionais no Novo Mercado do Brasil:

Uma Análise da Influência de Auditorias Big Four e não Big Four

prol de lucros futuros, ou seja, as empresas administram o seu resultado do período de modo a exibir melhores resultados no futuro.

O gerenciamento de resultado ocorre através do manejo formal das contas de resultado ou através de decisões ou atos concretos que causam implicações no fluxo de caixa das empresas (BARALDI; PEREIRA, 2011). A manipulação da informação contábil pode ocorrer pelo gerenciamento de resultados através das escolhas contábeis ou gerenciamento de resultados através das atividades.

\subsection{FORMAS DE GERENCIAMENTO (ACCRUALS E DECISÕES OPERACIONAIS)}

Martinez (2013) divide o gerenciamento de resultados em duas categorias: gerenciamento de resultados por accruals e o gerenciamento de resultados por decisões operacionais, as quais apresentam algumas diferenças fundamentais, entre elas o impacto no fluxo de caixa operacional.

Martinez (2001) afirma que não há empecilhos no registro de accruals, na verdade o intuito é mensurar o lucro no seu sentido econômico, aquele que representa acréscimo efetivo na riqueza patrimonial da unidade econômica, independentemente da movimentação financeira. A questão chave é saber até que ponto a discricionariedade intrínseca à contabilidade está sendo utilizada em favor do "bem informar" ou em benefício de grupos particulares, como gestores ou acionistas majoritários, que buscam a consecução de seus objetivos (GOULART, 2007).

O problema referente ao gerenciamento de resultado está no fato de o gestor discricionariamente aumentar ou diminuir esses accruals como objetivo de influenciar o lucro. Devido ao fato de que o gestor toma a decisão de aumentar ou diminuir os accruals por motivos alheios à realidade do negócio, cria-se a necessidade didática de subdividir estas em: Discricionários (seriam artificiais e teriam como único proposito "gerenciar" o resultado contábil) e Não Discricionários (de acordo como a realidade do negócio). Os accruals discricionários costumam ser utilizados como proxy para o Gerenciamento de Resultados nas pesquisas positivas.

Por outro lado, o gerenciamento de resultados por decisões operacionais, também mencionado na literatura internacional como gerenciamento de resultados reais (ROYCHOWDHURY, 2006; GUNNY, 2010; MARTINEZ, 2011) difere da manipulação pelos accruals por envolver atividades relacionadas ao negócio propriamente dito. Para Cupertino (2013), sabendo-se que a detecção da manipulação por atividades reais é mais difícil que a efetuada pelo gerenciamento por accruals, os gestores estruturam transações para atingir o nível desejado de lucros.

Roychowdhury (2006) define a manipulação das atividades reais como desvios das práticas operacionais normais, motivadas pelo desejo dos gestores em enganar usuários da informação em relação a certas metas de relatórios financeiros. Sendo assim, o gerenciamento de resultados por decisões operacionais ocorre quando os gestores manipulam as atividades operacionais da empresa para aumentar ou reduzir os resultados do período corrente (GUNNY, 2010). Vale destacar que, embora aparentemente exiba resultados a curto prazo, o gerenciamento por decisões operacionais pode reduzir o valor da empresa, porque as ações tomadas no período atual para aumentar os lucros podem ter um efeito negativo nos fluxos de caixa em períodos futuros (ROYCHOWDHURY, 2006).

Reis, Lamounier e Bressan (2015) encontram na literatura nacional e internacional diversas formas de manifestação do gerenciamento através de atividades reais: 1) aceleração do processo de vendas e/ou aumento dos descontos (temporários) sobre o preço ou diminuição das restrições de créditos aos clientes; 2) o momento de entrega dos produtos aos clientes; 3) aumento dos níveis de produção para redução dos custos dos produtos vendidos; 4) redução dos gastos com pesquisas e desenvolvimento, treinamento de pessoal, manutenção do parque fabril, 
Sena, Dias Filho e Moreira (2020)

Gerenciamento de Resultados por Decisões Operacionais no Novo Mercado do Brasil:

Uma Análise da Influência de Auditorias Big Four e não Big Four

despesas com vendas e administrativas, despesas com publicidade; e 5) o momento de realizar investimentos.

É possível encontrar modelos estatísticos que buscam evidenciar o nível de gerenciamento de resultado por decisões operacionais a partir de eventos como os listados no parágrafo anterior. Roychowdhury (2006), com base na pesquisa desenvolvida por Dechow, Kothari e Watts (1998), desenvolveu modelos estatísticos para capturar o nível de gerenciamento de resultado por manipulação nas vendas, gastos discricionários e produção. Anderson, Banker e Janakiraman (2003) apresentam um modelo para verificar o comportamento através das despesas de vendas, gerais e administrativas.

Zang (2011) buscou verificar se os gestores utilizam o gerenciamento de resultados por accruals e por decisões operacionais como substitutos entre si. Utilizando um modelo empírico que incorpora os custos associados aos dois métodos de gerenciamento de resultados e capta as decisões subsequentes dos gerentes, o autor confirma sua hipótese inicial. Quando o gerenciamento de resultados por accruals é restringido devido a implementação da SOX, as empresas usam a manipulação de atividades reais para manipular os resultados.

Nesse mesmo sentido, Cupertino (2013) tem como um dos objetivos constatar se a adoção do IFRS teve impacto no nível de manipulação das estratégias de gerenciamento. Seus resultados apontam que, após a implantação do IFRS, o nível de manipulação por accruals diminuiu, enquanto por decisões operacionais aumentou. Esse resultado fundamenta-se no fato de os padrões de convergência das regras contábeis às normas internacionais restringir o grau de discricionariedade do gestor para a manipulação dos resultados por accruals.

Confirmada a prática de gerenciamento de resultados por decisões operacionais em empresas brasileiras, os estudos sugerem que pesquisas futuras busquem identificar fatores que exercem influência no nível de gerenciamento de resultados (CARDOSO; MARTINEZ, 2009; SANTOS et al., 2011; SILVA, 2015). Esses fatores podem servir como incentivadores ou inibidores das práticas de gerenciamento de resultado, podendo destacar como exemplo a presença de regulação no setor, conselho de administração, nível de governança corporativa, qualidade da auditoria independente, entre outros. Silva (2015) sugere ainda que pesquisas busquem identificar, entre os instrumentos de governança, quais são mais eficientes para inibir o gerenciamento de resultado.

\subsection{AUDITORIA INDEPENDENTE COMO FATOR INIBIDOR DE GERENCIAMENTO DE RESULTADOS}

Healy e Palepu (2001) mencionam diversos recursos para mitigar os problemas de assimetria e conflito. Destaca-se dentre os exemplos o papel dos intermediários informacionais (analistas de mercado, agências de classificação, mídia especializada, auditores independentes), que sua atuação tem como consequência a redução da assimetria informacional (RAMOS; MARTINEZ, 2006; MARTINEZ, 2011). Esses intermediários agem tanto no sentido de "divulgar" informação privada dos insiders, quanto ao problema de agência, na medida em que permitem o monitoramento das tentativas dos agentes de expropriar os principais.

A auditoria independente é considerada um mecanismo de governança corporativa, pois busca reduzir o problema de assimetria informacional e incentivos conflitantes entre insiders e outsiders (BRAUNBECK, 2010). Em outras palavras, Jensen e Mackelin (1976) afirmam que a auditoria independente busca a minimização dos custos de agência resultantes dos conflitos nas relações entre principal e agente. Sendo assim a auditoria tem como objetivo assegurar que que a informação financeira que foi auditada é razoavelmente livre de erros ou parcialidade e reflete de maneira fidedigna os eventos e fatos econômicos da organização.

Watts e Zimmerman (1986) propuseram a hipótese da auditoria com base no problema de informação delineado por Akerlof (1970) e também nas questões de agência desenvolvidas 
Gerenciamento de Resultados por Decisões Operacionais no Novo Mercado do Brasil: Uma Análise da Influência de Auditorias Big Four e não Big Four

por Jensen e Meckling (1976). Sob esta hipótese, dado o caráter maximizador dos principais, na busca por eficiência contratual, estes estariam dispostos a incorrer em atividades de monitoramento que se apresentassem capazes de reduzir os custos de agência. Nesta conjuntura a auditoria representa atividade de monitoramento relacionada à produção e divulgação da informação contábil. Watts e Zimmerman (1986) argumentaram que, caso fosse esta uma hipótese razoável, haveria de se observar atividades de monitoramento similares à auditoria em qualquer contexto no qual esteja presente o conflito de agência e assimetria informacional, em qualquer tempo.

O "valor" de uma auditoria para os usuários das demonstrações contábeis existirá no contexto do relacionamento de agência e na medida em que a percepção dos usuários seja a probabilidade de que o auditor reporte de fato uma quebra contratual é não nula. Existirá, por tanto, demanda por auditoria se a probabilidade de que o auditor informe a desconformidade for não nula (WATTS; ZIMMERMAN, 1986). Martinez (2011) afirma que a relação entre as empresas auditadas e as firmas de auditoria também sofrem com conflitos de agência, ao passo que essas possuem grande preocupação em perder seus clientes, sendo esse problema mais grave quando a empresa de auditoria for menor. Sendo assim, as maiores firmas de auditoria, por possuírem maior independência, estão mais propensas a questionar as demonstrações contábeis de seus clientes. Dessa maneira, DeAngelo (1981) considerou que a probabilidade de um auditor informar uma quebra contratual, mediante a existência de uma desconformidade, depende da probabilidade de o auditor: 1) descobrir a desconformidade existente Competência; e 2) informar de fato a desconformidade descoberta - Independência.

No Brasil, o primeiro trabalho a investigar a relação da Auditoria Independente com o gerenciamento de resultado foi a tese de doutorado de Martinez (2001) em seu apêndice A. O autor tinha como hipótese que quanto mais independente for a firma de auditoria da gestão, menor o nível de gerenciamento de resultados da empresa auditada. Para o período analisado (1995 a 1999, anterior ao escândalo envolvendo a Arthur Andersen) foi utilizado o grupo composto pelas cinco maiores empresas de auditoria no mundo (BigFive) - Andersen, Deloitte, PricewaterhouseCoopers, Ernst \& Young e KPMG - como mais independente em oposição às firmas nacionais. Foi utilizado o teste de hipóteses de diferença de médias entre os accruals discricionários das empresas brasileiras de capital aberto, concluindo-se que empresas auditadas por firmas nacionais possuem maior média de accruals discricionárias.

Em 2011 o mesmo autor elabora uma nova pesquisa para investigar a influência da Governança Corporativa no nível de gerenciamento de resultado por escolhas contábeis (accruals discricionários) e decisões operacionais em empresas brasileiras de capital aberto durante o período de 1998 a 2004. Sua hipótese referente às firmas de auditoria permaneceu a mesma, obtendo a conclusão que a listagem em segmentos especiais e a auditoria por Big Four reduzem o gerenciamento de resultados por escolhas contábeis. Entretanto, esses mesmos fatores não minimizam o gerenciamento por decisões operacionais.

Almeida e Almeida (2009) investigam a capacidade das firmas de auditoria em mitigar o gerenciamento de resultado em empresas de capital aberto no Brasil no período de 1999 a 2005. Através da utilização do modelo KS para estimação dos accruals discricionários e testes de hipóteses não paramétricos, os resultados indicam que as empresas auditadas pelas Big Four possuem menor grau de accruals discricionários em relação às demais, confirmando a hipótese acerca da capacidade para mitigar práticas de gerenciamento de resultado.

Santana et al (2014) verificaram se os níveis de gerenciamento de resultado são diferentes quando a empresa é auditada por uma firma de auditoria Big Four ou não Big Four. Para isso, utilizaram o método de medida de gerenciamento de resultado agregado (Aggregate Measure of Earnings Management) baseado em Leuz, Nanda e Wysocki (2003). Os autores concluiram que quanto maior a firma de auditoria menor será o nível de Gerenciamento de 
Gerenciamento de Resultados por Decisões Operacionais no Novo Mercado do Brasil: Uma Análise da Influência de Auditorias Big Four e não Big Four

Resultados na empresa auditada, devido a seu maior grau de independência e competência técnica. A amostra foi composta por 329 empresas listadas na B3 e concluiu-se que não há diferença significativa entre os níveis de gerenciamento de resultado entre as empresas auditadas por firmas Big Four ou não Big Four.

Queiroz (2016) utilizou o modelo Jones Modificado de Dechow et al. (1995) para estimar o nível de gerenciamento por meio de accruals discricionários nas empresas brasileiras de capital aberto no período de 2011 a 2014. Os resultados obtidos apontam que não há diferença estatisticamente significante entre as firmas de auditoria, permitindo afirmar que o tipo de firma (Big Four ou não Big Four) não impacta no nível dos accruals discricionários.

Silva et al (2016) analisou a influência da auditoria sobre o gerenciamento de resultados por accruals discricionários utilizando o modelo KS em uma amostra compreendida por 106 empresas listadas no Segmento Novo Mercado da B3, no período de 2010 a 2012. Os resultados demonstraram que as empresas de auditoria Big Four possuem relação negativa com o gerenciamento para o aumento do resultado por meio de accruals nas empresas brasileiras.

\section{METODOLOGIA}

Para o desenvolvimento inicial desta pesquisa, foram realizadas pesquisas exploratórias de fontes bibliográficas, desenvolvidas com a intenção de promover uma visão geral acerca do tema estudado, além de apurar como a literatura atual o aborda. Em seguida, através de pesquisas natureza quantitativa, para medir numericamente as hipóteses levantadas, foi analisada a relação descrita como problemática desse trabalho.

\subsection{COLETA E ANÁLISE DE DADOS}

Para o levantamento de dados, no campo da pesquisa descritiva, foi realizada uma pesquisa documental e análise de dados através de modelos de regressão do método de Mínimos Quadrados Generalizados (FGLS). A amostra da pesquisa foi composta por empresas brasileiras de capital aberto listadas no Novo Mercado da B3. A opção por limitar a amostra às empresas do Novo Mercado se vale de que o nível de governança corporativa é um fator inibidor de gerenciamento de resultados. Portanto, essa ação tem por objetivo não viesar o nível anormal das proxies estudadas. As informações serão obtidas de forma secundária através do Software Economática®.

Devido a características próprias do setor financeiro e de seguros, as empresas pertencentes a esse foram excluídas da população da pesquisa. Foram excluídas, ainda, as observações que não possuíam dados necessários para a análise desta pesquisa. Dessa maneira, a amostra total constava de 515 observações para o período de 2012 a 2017, sendo 465 empresas-ano auditadas por Big Four e 50 por não Big Four.

Aponta-se Big Four como o grupo das quatro maiores firmas de auditoria no mundo, sendo composta por: Deloitte, Ernst \& Young, KPMG e PriceWaterhouse Coopers. Essas empresas possuem reconhecimento devido aos fatores de qualidade de auditoria (competência e independência), fazendo com que estar ou não presente nesse grupo seja considerado na literatura como uma proxy para diversas variáveis como: qualidade da auditoria independente, independência da auditoria independente, tamanho da firma de auditoria, entre outras. Nesse estudo optou-se não usar esses rótulos, mas apenas verificar a presença ou ausência nesse grupo para mensurar a variável utilizada.

O período escolhido para análise da amostra foi compreendido entre os anos de 2010 a 2017 por ser, respectivamente, o ano de início da obrigatoriedade das normas IFRS para as empresas brasileiras de capital aberto e o último ano com dados disponíveis. Entretanto não utilizou-se as observações dos períodos de 2010 e 2011, apenas algumas informações para alimentar as variáveis dos anos seguintes, pois para o cálculo de uma das regressões era 
Gerenciamento de Resultados por Decisões Operacionais no Novo Mercado do Brasil: Uma Análise da Influência de Auditorias Big Four e não Big Four

necessário a informação de dois períodos anteriores (t-2). Optou-se pelo período pós-IFRS devido à reduzida quantidade de trabalhos que se propõem a investigar o gerenciamento de resultado por decisões operacionais nesse período no Brasil. Cupertino (2013) afirma que o nível de manipulação por accruals diminuiu, enquanto por decisões operacionais aumentou após a implantação do IFRS, fato que pode ser atribuído às restrições impostas pelas normas de harmonização internacional. Outros trabalhos concluem que a preferência dos administradores pela manipulação do resultado pelo gerenciamento por accruals mudou após diversos escândalos financeiros como Enron e WorldCom, fazendo com que as empresas optassem pelo gerenciamento através de decisões operacionais (GRAHAM; HARVEY; RAJGOPAL, 2005; COHEN; DEY; LYS, 2008).

As observações foram organizadas por dados em painel de efeitos aleatórios e foi utilizado o método dos Mínimos Quadrados Generalizados (FGLS) para definição dos coeficientes, uma vez que se mostra robusto diante de problemas de heterocedasticidade e/ou autocorrelação.

A variável dependente desse estudo (nível de gerenciamento de resultado por decisões operacionais) foi operacionalizada através da proxy obtida a partir dos resíduos dos modelos baseados em Roychowdhury (2006) para cada hipótese apresentada.

$$
\begin{aligned}
& \frac{F C O_{i t}}{A T_{i t-1}}=\beta_{0}+\beta_{1}\left(\frac{1}{A T_{i t-1}}\right)+\beta_{2}\left(\frac{R V_{i t}}{A T_{i t-1}}\right)+\beta_{3}\left(\frac{\Delta R V_{i t}}{A T_{i t-1}}\right)+\varepsilon_{\mathrm{it}} \\
& \frac{D V G A_{i t}}{A T_{i t-1}}=\beta_{0}+\beta_{1}\left(\frac{1}{A T_{i t-1}}\right)+\beta_{2}\left(\frac{R V_{i t-1}}{A T_{i t-1}}\right)+\varepsilon_{\mathrm{it}} \\
& \frac{P R O D_{i t}}{A T_{i t-1}}=\beta_{0}+\beta_{1}\left(\frac{1}{A T_{i t-1}}\right)+\beta_{2}\left(\frac{R V_{i t}}{A T_{i t-1}}\right)+\beta_{3}\left(\frac{\Delta R V_{i t}}{A T_{i t-1}}\right)+\beta_{4}\left(\frac{\Delta R V_{i t-1}}{A T_{i t-1}}\right)+\varepsilon_{\mathrm{it}}
\end{aligned}
$$

Onde cada variável faz menção a empresa i:

$\mathrm{FCO}_{\mathrm{t}}=$ Fluxo de Caixa Operacional no período t;

DVGA $_{\mathrm{t}}=$ Despesas com Vendas, Gerais e Administrativas no período t;

PROD $_{\mathrm{t}}=$ Custo do Produto Vendido + Variação dos Estoques no período t;

$\mathrm{AT}_{\mathrm{t}-1}=$ Ativo Total do período $\mathrm{t}-1$;

$\mathrm{RV}_{\mathrm{t}}=$ Receita de Vendas no periodo $\mathrm{t}$;

$\Delta \mathrm{RV}_{\mathrm{t}}=$ Variação na Receita de Vendas do período $\mathrm{t}$ em relação ao $\mathrm{t}-1$;

$\Delta \mathrm{RV}_{\mathrm{t}-1}=$ Variação na Receita de Vendas do período $\mathrm{t}-1$ em relação ao $\mathrm{t}-2$;

\subsection{HIPÓTESES DE PESQUISA E DETERMINAÇÃO DOS MODELOS ECONOMÉTRICOS}

Cada hipótese mencionada abaixo foi baseada na premissa de que as firmas de auditoria pertencentes ao grupo Big Four, por possuir maior independência quando comparada com as demais, tolerem menos o gerenciamento de resultados das empresas auditadas (MARTINEZ, 2001). Sendo assim, as empresas que gerenciam os resultados contábeis para aumentá-los apresentarão CFO e/ou DVGA baixos e PROD altos (ROYCHOWDHURY, 2006; MARTINEZ; CARDOSO, 2009; GUNNY, 2010; ZANG, 2012; CUPERTINO, 2013; SILVA, 2015; REIS; LAMOUNIER; BRESSAN, 2015). Entretanto, como é de conhecimento que o gerenciamento de resultado pode resultar tanto no aumento quanto redução dos lucros contábeis, esse trabalho se propõe a analisar apenas o valor dos níveis anormais das decisões operacionais, desconsiderando seu sinal. 
Gerenciamento de Resultados por Decisões Operacionais no Novo Mercado do Brasil: Uma Análise da Influência de Auditorias Big Four e não Big Four

O gerenciamento de resultado por decisões operacionais pode acontecer através da manipulação de vendas. Roychowdhury (2006) define a manipulação de vendas como tentativas dos gerentes de aumentar temporariamente as vendas durante o ano, através de descontos nos preços ou condições de crédito mais brandas. Sendo assim, apresenta-se a primeira hipótese do estudo:

H1: As empresas brasileiras auditadas por Big Four apresentam menor fluxo de caixa operacional anormal comparado com as empresas brasileiras auditadas por não Big Four.

O modelo que contempla as despesas discricionárias analisa as variações nas contas de despesas de vendas, gerais, administrativas, incluindo nessas as despesas comerciais, com pesquisa e desenvolvimento $(\mathrm{P} \& \mathrm{D})$, publicidade e propaganda, etc. Espera-se que a variação desse tipo de despesa acompanhe as variações nas Receitas de Venda de forma diretamente proporcional (MARTINEZ; CARDOSO, 2009). Sendo assim, variações anormais (expressas pelos resíduos da regressão 2) indica a ocorrência de gerenciamento de resultados através de manipulação das contas vinculadas a DVGA (SILVA, 2015). A hipótese 2 relaciona o nível de gerenciamento de resultados por meio de DVGA com as firmas de auditoria:

H2: As empresas brasileiras auditadas por Big Four apresentam valores anormais menores em despesas com vendas, gerais e administrativas comparado com as empresas brasileiras auditadas por não Big Four.

Para Silva (2015), o modelo de produção anormal baseia-se na premissa de que o comportamento dos custos de produção (custo das vendas e variação de estoques) está relacionado com a receita e suas variações no decorrer dos anos. Martinez e Cardoso (2009) afirmam que um aumento anormal da produção proporciona a redução de custos unitários de produção, uma vez que os custos fixos são rateados por uma maior quantidade de unidades. Dessa forma, o custo dos produtos vendidos se tornam menores, repercutindo positivamente no resultado contábil do período. Diante do exposto, a terceira hipótese da pesquisa afirma que:

H3: As empresas brasileiras auditadas por Big Four apresentam menores níveis anormais de produção comparado com as empresas brasileiras auditadas por não Big Four.

O teste de hipóteses foi realizado de forma similar aos modelos utilizados por Roychowdhury (2006), Gunny (2010), Zang (2012) e Cupertino (2013), apresentados a seguir:

$$
\begin{aligned}
& A F C O_{i t}=\beta_{0}+\beta_{1} A U D I T_{i t}+\beta_{2} T_{A} M_{i t}+\beta_{3} M T B_{i t}+\beta_{4} R O A_{i t}+\varepsilon_{\mathrm{it}} \\
& A D V G A_{i t}=\beta_{0}+\beta_{1} A U D I T_{i t}+\beta_{2} T_{A} M_{i t}+\beta_{3} M T B_{i t}+\beta_{4} R O A_{i t}+\varepsilon_{\mathrm{it}} \\
& A_{P R O D}=\beta_{0}+\beta_{1} A U D I T_{i t}+\beta_{2} T_{A} M_{i t}+\beta_{3} M T B_{i t}+\beta_{4} R O A_{i t}+\varepsilon_{\text {it }}
\end{aligned}
$$

Onde cada variável faz menção a empresa i:

$\mathrm{AFCO}_{\mathrm{t}}=$ Nível anormal de Fluxo de Caixa Operacional no período $t$;

$\mathrm{ADVGA}_{\mathrm{t}}=$ Nível anormal de Despesas com Vendas, Gerais e Administrativas no período $\mathrm{t}$

$\mathrm{APROD}_{\mathrm{t}}=$ Nível anormal de Custo do Produto Vendido + Variação dos Estoques no período t;

$\mathrm{AUDIT}_{\mathrm{t}}=$ Variável dummy que assume 1 para empresas auditadas por Big Four e 0 caso contrário no período t;

$\mathrm{TAM}_{\mathrm{t}}=$ Logaritmo natural do Ativo Total (Tamanho) no período t; 
Gerenciamento de Resultados por Decisões Operacionais no Novo Mercado do Brasil: Uma Análise da Influência de Auditorias Big Four e não Big Four período t;

$\mathrm{MTB}_{\mathrm{t}}=$ Razão entre o valor de mercado e o Patrimônio Líquido (Market-to-Book) no

$\mathrm{ROA}_{\mathrm{t}}=$ Razão entre o Lucro Líquido e Ativo Total (Retorno sobre o Ativo) no período $\mathrm{t}$

A variável independente principal foi operacionalizada através da dummy AUDIT, a qual expressa se a empresa é auditada por Big Four ou não Big Four. Espera-se que o coeficiente $\beta$ associado a essa variável tenha valor negativo e significativo para as três hipóteses apresentadas, revelando assim uma relação inversamente proporcional da firma de auditoria em relação ao nível de gerenciamento de resultados por decisões operacionais.

Destaca-se a utilização de variáveis de controle, utilizadas para aumentar a validade interna da pesquisa ao identificar outros possíveis fatores que influenciam a variável dependente além das variáveis principais. Esse estudo seguiu o mesmo padrão adotado por Roychowdhury (2006), Gunny (2010), Zang (2012) Cupertino (2013), Silva (2015) e Reis, Lamounier e Bressan (2015), inserindo variáveis de controle para o tamanho da empresa, oportunidade de crescimento e desempenho. De forma individual, essas condições podem influenciar o nível do gerenciamento de resultados, sendo medidas, respectivamente, através do logaritmo natural do ativo total (TAM), índice Market-to-book (MTB) e rentabilidade do ativo (ROA).

\section{ANÁLISE DOS RESULTADOS}

\subsection{ESTATÍSTICA DESCRITIVA}

A tabela 1 apresenta os resultados estatísticos para cada variável utilizada nas três primeiras equações deste trabalho.

Tabela 1 - Estatística Descritiva

\begin{tabular}{|c|c|c|c|c|c|c|c|c|}
\hline Variáveis & $\begin{array}{l}\mathbf{F C O} / \\
\mathbf{A T}_{\text {it-1 }}\end{array}$ & $\begin{array}{c}\text { DVGA/ } \\
\text { AT }_{\text {it-1 }} \\
\end{array}$ & $\begin{array}{c}\text { PROD/ } \\
\text { AT }_{\text {it-1 }} \\
\end{array}$ & $\begin{array}{c}\mathbf{1} / \\
\mathbf{A T}_{\text {it-1 }}\end{array}$ & $\begin{array}{l}\mathbf{R} \mathbf{V}_{\mathrm{it} /} / \\
\mathbf{A T _ { \text { it } - 1 }}\end{array}$ & $\begin{array}{c}\mathbf{R V}_{\text {it }-1 /} \\
\mathbf{A T}_{\mathrm{it}-1} \\
\end{array}$ & $\begin{array}{c}\Delta \mathrm{RV}_{\mathrm{it}} / \\
\mathrm{AT}_{\mathrm{it}-1}\end{array}$ & $\begin{array}{c}\Delta V_{\mathrm{it}-1 /} \\
\mathrm{AT}_{\mathrm{it}-1} \\
\end{array}$ \\
\hline Média & 0.0684 & 0.1570 & 0.5337 & 0.00000055 & 0.7388 & 0.6976 & 0.0412 & 0.0447 \\
\hline Erro & 0.0036 & 0.0061 & 0.0224 & 0.00000004 & 0.0260 & 0.0236 & 0.0060 & 0.0062 \\
\hline Mediana & 0.0637 & 0.1058 & 0.3884 & 0.00000027 & 0.5757 & 0.5258 & 0.0304 & 0.0382 \\
\hline Desvio padr & 0.0806 & 0.1389 & 0.5092 & 0.00000096 & 0.5892 & 0.5357 & 0.1353 & 0.1408 \\
\hline Variâ & 0.0065 & 0.0193 & 0.2593 & 0.00000000 & 0.3472 & 0.2869 & 0.0183 & 0.0198 \\
\hline Intervalo & 0.8477 & 0.8284 & 3.8515 & 0.00001078 & 4.1494 & 3.7390 & 1.6290 & 1.5762 \\
\hline Mínimo & -0.2624 & -0.0090 & -0.0271 & 0.00000000 & -0.0137 & -0.0182 & -0.4210 & -0.5132 \\
\hline Máximo & 0.5853 & 0.8194 & 3.8243 & 0.00001078 & 4.1357 & 3.7207 & 1.2080 & 1.0630 \\
\hline
\end{tabular}

Fonte: Elaborado pelo autor (2019).

Através dessa tabela, destaca-se que, para todas as variáveis utilizadas nas equações citadas, a média tem valor inferior a 1 (um), pelo fato de todas as variáveis apresentarem o AT it-1 como denominador. Nota-se ainda que a média de RV e $\Delta \mathrm{RV}$ de diferentes períodos possuem valores próximos.

É apresentada a matriz de correlação através da tabela 2, com o intuito de identificar se há existência de alta correlação entre variáveis independentes para os modelos econométricos para definição do nível normal de FCO, DVGA e PROD. 
Sena, Dias Filho e Moreira (2020)

Gerenciamento de Resultados por Decisões Operacionais no Novo Mercado do Brasil: Uma Análise da Influência de Auditorias Big Four e não Big Four

Tabela 2 - Matriz de Correlação

\begin{tabular}{|c|c|c|c|c|c|c|c|c|}
\hline Variáveis & $\begin{array}{l}\mathbf{F C O} / \\
\mathbf{A T}_{\text {it-1 }}\end{array}$ & $\begin{array}{c}\text { DVGA/ } \\
\text { AT }_{\text {it }-1} \\
\end{array}$ & $\begin{array}{c}\text { PROD/ } / \\
\text { AT }_{\text {it-1 }} \\
\end{array}$ & $\begin{array}{c}\mathbf{1} / \\
\mathbf{A T}_{\text {it-1 }}\end{array}$ & 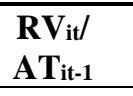 & $\begin{array}{l}\mathbf{R V} \mathbf{i t}_{\text {-1/ }} \\
\mathbf{A T}_{\text {it-1 }}\end{array}$ & $\begin{array}{l}\Delta \mathrm{RV}_{\mathrm{it} /} / \\
\mathbf{A T}_{\mathrm{it}-1}\end{array}$ & $\begin{array}{c}\Delta \mathrm{RV}_{\mathrm{it}-1 /} / \\
\mathrm{AT}_{\mathrm{it}-1} \\
\end{array}$ \\
\hline FCO/AT it-1 & 1 & & & & & & & \\
\hline DVGA/AT it-1 & 0.2768 & 1 & & & & & & \\
\hline PROD/AT it-1 & 0.0888 & 0.2954 & 1 & & & & & \\
\hline $1 / \mathrm{AT}_{\mathrm{it}-1}$ & 0.0783 & 0.1793 & -0.0238 & 1 & & & & \\
\hline $\mathbf{R} \mathbf{V}_{\mathrm{it}} / \mathbf{A T}_{\mathrm{it}-\mathbf{1}}$ & 0.2190 & 0.5166 & 0.9579 & -0.0061 & 1 & & & \\
\hline $\mathbf{R} \mathbf{V}_{\mathrm{it}-1} / \mathbf{A} \mathbf{T}_{\mathrm{it}-1}$ & 0.1770 & 0.5019 & 0.9423 & 0.0147 & 0.9755 & 1 & & \\
\hline$\Delta R V_{i t} / A T_{i t-1}$ & 0.2522 & 0.2623 & 0.4404 & -0.085 & 0.4923 & 0.2889 & 1 & \\
\hline$\Delta R V_{\mathrm{it}-1} / \mathbf{A T}_{\mathrm{it}-1}$ & 0.1796 & 0.2611 & 0.4398 & -0.068 & 0.4907 & 0.4486 & 0.3605 & 1 \\
\hline
\end{tabular}

Percebe-se uma alta correlação (> 0,9) entre as variáveis PROD, RV it e RV it-1, entretanto não inviabiliza (multicolinearidade) a execução dos modelos de estimação por regressão, uma vez que PROD é uma variável dependente, e as variáveis de RV de diferentes períodos não estão presentes simultaneamente na mesma equação. A forte correlação entre PROD e RV para o mesmo período e o período anterior revela que o aumento nas Receitas Liquidas de Venda é seguido pelo aumento no nível de Produção (Custos dos Produtos Vendidos e Variação de Estoque), já que as empresas, para ter maior disponibilidade de produtos à venda, precisam produzir mais e consequentemente aumentar os custos totais de produção. (REIS; LAMOUNIER; BRESSAN, 2015)

\subsection{ESTIMAÇÃO DOS VALORES ANORMAIS}

O nível anormal de FCO, DVGA e PROD deve ser calculado através da diferença entre o valor esperado e o valor observado para cada variável dependente. Dessa maneira, a tabela 3 apresenta os estimadores para o nível normal das variáveis dependentes das equações 1,2 e 3 .

Tabela 3 - Estimadores para definição do Nível Anormal da variável dependente

\begin{tabular}{|c|c|c|c|}
\hline Variáveis & Equação (1) & Equação (2) & Equação (3) \\
\hline Intercepto & $0.034805 * * *$ & $0.056252 * * *$ & $-0.040152 *$ \\
\hline $1 / \mathrm{AT}_{\mathrm{it}-1}$ & 7789.9 . & $37103 \quad * * *$ & -4634.6 \\
\hline $\mathbf{R V}_{\mathrm{it}} / \mathbf{A T}_{\mathrm{it}-1}$ & $0.030204 * *$ & & 0.7925 \\
\hline $\mathbf{R V}_{\text {it-1/1 }} / \mathbf{A T}_{\text {it-1 }}$ & & $0.111080 * * *$ & \\
\hline$\Delta R V_{\text {it }} / A_{\text {it-1 }}$ & $0.065621 * *$ & & 0.011096 \\
\hline$\Delta R V_{\text {it-1 }} / \mathbf{A T}_{\mathrm{it}-1}$ & & & $-0.045864 *$ \\
\hline Múltiplo $\mathbf{R}^{2}$ & 0.072489 & 0.26885 & 0.91615 \\
\hline
\end{tabular}

Fonte: Elaborado pelo autor (2019).

Legenda: Índices de Significância: $* * *=0.001 ; * *=0.01 ; *=0.05 ; .=0.1$

No modelo de regressão para determinação do nível normal de FCO, todos os estimadores das variáveis independentes se mostram positivos e significantes (1/AT $\mathrm{T}_{\mathrm{it}-1}$, ao nível de 10\%; as demais, de 1\%). Ou seja, um aumento na Receita Líquida de Vendas e/ou na sua Variação provoca um aumento nos Fluxos de Caixa Operacional. Esses resultados corroboram com os achados na literatura atual, entre eles: Roychowdhury (2006) e Cupertino (2013). Diferente desses autores, dos Reis, Lamounier e Bressan (2015) utilizam a variável o $\Delta \mathrm{RV}$ it-1 em substituição a $\Delta R V_{\text {it }}$ e não encontram significância estatística.

Em relação aos estimadores para a equação (2), todos coeficientes apresentam significância estatística ao nível de $0,1 \%$ e valores positivos. Sendo assim, espera-se um 
Gerenciamento de Resultados por Decisões Operacionais no Novo Mercado do Brasil: Uma Análise da Influência de Auditorias Big Four e não Big Four

aumento nas Despesas de Vendas, Gerais e Administrativas quando houver aumento na Receita Líquida de Vendas. Os mesmos autores citados no parágrafo anterior e Zang (2012) encontram resultados similares a esses apresentados.

$\mathrm{Na}$ equação (3) para cálculo do nível normal para PROD, o sinal dos estimadores corrobora integralmente os estudos de Roychowdhury (2006), Cupertino (2013) e Zang (2012), diferindo de Martinez e Cardoso (2009) apenas em $\Delta \mathrm{RV}$ it-1. Entretanto, o coeficiente para $\Delta \mathrm{RV}_{\text {it }}$ não apresenta significância estatística nesse estudo, diferentemente dos demais.

A partir da definição desses estimadores, foi possível determinar a diferença entre o valor esperado e observado para cada variável dependente (FCO, DVGA e PROD), obtendo assim os valores anormais respectivos: AFCO, ADVGA e APROD. Essas novas variáveis serão utilizadas nas regressões seguintes como proxy para o nível de gerenciamento de resultado por meio de decisões operacionais, avaliando assim a influência da empresa de auditoria (Big Four ou não Big Four) como inibidora de manipulação de resultados.

\subsection{TESTE DAS HIPÓTESES}

Neste item serão apresentados os modelos estatísticos para análise das hipóteses dessa pesquisa, de forma geral, objetivando verificar se há diferença no nível de gerenciamento de resultados através decisões operacionais das empresas brasileira do novo mercado quando auditadas por Big Four ou não Big Four.

A primeira hipótese (H1) afirma que as empresas citadas, quando auditadas por Big Four, apresentam menores níveis anormais de Fluxo de Caixa Operacional. A tabela 4 apresenta os resultados para a equação (4).

Tabela 4 - Regressão da Equação (4)

\begin{tabular}{|c|c|c|c|c|}
\hline Variáveis & Estimativa & Desvio Padrão & Valor $\mathbf{z}$ & Teste p \\
\hline Intercepto & 0.0457021 & 0.1191818 & 0.3835 & 0.701375 \\
\hline AUDIT & -0.0219478 & 0.0210702 & -1.0417 & 0.297574 \\
\hline TAM & 0.0035125 & 0.0053450 & 0.6572 & 0.511082 \\
\hline МTB & 0.0074469 & 0.0027301 & 2.7277 & $0.006377 * *$ \\
\hline ROA & -0.0119562 & 0.0397939 & -0.3005 & 0.763831 \\
\hline Múltiplo R $\mathbf{R}^{2}$ & 0,0112 & & & \\
\hline Teste Hausman & 16.989 & & & \\
\hline (p) & $(0.0019)$ & & & \\
\hline Teste Durbin-Watson & 2.0694 & & & \\
\hline (p) & $(0.7864)$ & & & \\
\hline Teste Breusch Pagan & 38.7 & & & \\
\hline (p) & $(0.0000)$ & & & \\
\hline
\end{tabular}

Fonte: Elaborado pelo autor (2019).

Legenda: Índices de Significância: $* * *=0.001 ; * *=0.01 ; *=0.05 ;=0.1$

Pode-se perceber que para a amostra em destaque, apenas a variável Market-to-Book apresenta significância estatística (a um nível de 1\%), possuindo uma relação positiva com o nível anormal de FCO. Esse resultado contraria o encontrado por dos Reis, Lamounier e Bressan (2015) no mercado nacional para o período de 2008 a 2013, mas corrobora com Roychowdhury (2006).

Quando da interpretação do estimador para AUDIT, o sinal negativo indicaria que a auditoria realizada por Big Four reduziria o nível anormal de FCO, logo o gerenciamento de resultados por decisões operacionais. Entretanto, o valor se mostra não significante estatisticamente, podendo ser considerado como zero. Nesse caso, não há diferença entre o nível de gerenciamento de resultado para empresas auditadas por Big Four ou não Big Four, refutando assim a hipótese 1 . 
Sena, Dias Filho e Moreira (2020)

Gerenciamento de Resultados por Decisões Operacionais no Novo Mercado do Brasil:

Uma Análise da Influência de Auditorias Big Four e não Big Four

A tabela 5 apresenta o resultado para a regressão da equação (5), que busca verificar a segunda hipótese da pesquisa, utilizando como proxy os valores anormais para Despesas de Venda, Gerais e Administrativas.

Tabela 5 - Regressão da Equação (5)

\begin{tabular}{|c|c|c|c|c|}
\hline Variáveis & Estimativa & Desvio Padrão & Valor z & Teste p \\
\hline Intercepto & 0.1789841 & 0.0856264 & 2.0903 & $0.03659 *$ \\
\hline AUDIT & 0.0121298 & 0.0121700 & 0.9967 & 0.31891 \\
\hline TAM & -0.0056407 & 0.0038480 & -1.4659 & 0.14268 \\
\hline MTB & 0.0100850 & 0.0013600 & 7.4155 & $0,00000 * * *$ \\
\hline ROA & -0.0910418 & 0.0185019 & -4.9207 & $0,00000 * * *$ \\
\hline Múltiplo $\mathbf{R}^{2}$ & 0.2179 & & & \\
\hline Teste Hausman & 72.961 & & & \\
\hline (p) & $(0.0000)$ & & & \\
\hline Teste Durbin-Watson & 1.6894 & & & \\
\hline (p) & $(0.0002)$ & & & \\
\hline Teste Breusch Pagan & 336.95 & & & \\
\hline (p) & $(0.0000)$ & & & \\
\hline
\end{tabular}

Nesse caso, o coeficiente para o Tamanho da empresa se mostrou novamente não significante. Logo não influencia o nível anormal de DVGA. Os índices de Market-to-Book e Retorno sobre Ativo apresentam significância estatística, porém influenciam em sentido contrários o ADVGA: o primeiro positiva, o segundo negativamente.

Diferentemente da equação (4), o coeficiente para AUDIT apresentou valor positivo, fato que indicaria uma influência para aumentar o nível anormal de DVGA quando auditada por Big Four. Porém, novamente esse coeficiente apresenta um valor não significativo estatisticamente, contrariando $\mathrm{H} 2$, ao passo que não há diferença no nível de gerenciamento de resultado por DVGA quando auditada por Big Four ou não Big Four.

A terceira e última hipótese considera o nível anormal de produção (APROD) como proxy para o gerenciamento de resultados, com indicação similar as demais para a influência da Big Four na inibição de manipulações. A tabela 6 apresenta os valores para a última regressão deste estudo

Tabela 6 - Regressão da Equação (6)

\begin{tabular}{|c|c|c|c|c|}
\hline Variáveis & Estimativa & Desvio Padrão & Valor $\mathrm{z}$ & Teste $p$ \\
\hline Intercepto & 0.1959818 & 0.1109139 & 1.7670 & 0.07723 \\
\hline AUDIT & -0.0011553 & 0.0142109 & -0.0813 & 0.93520 \\
\hline TAM & -0.0056315 & 0.0049932 & -1.1278 & 0.25939 \\
\hline МTB & 0.0106351 & 0.0016713 & 6.3632 & $0,000000 * * *$ \\
\hline ROA & 0.0191333 & 0.0214293 & 0.8929 & 0.37193 \\
\hline Múltiplo R ${ }^{2}$ & 0.1974 & & & \\
\hline Teste Hausman & 69.804 & & & \\
\hline (p) & $(0.0000)$ & & & \\
\hline $\begin{array}{l}\text { Teste Durbin-Watson } \\
\text { (p) }\end{array}$ & 1,3945 & & & \\
\hline Teste Breusch Pagan & 228.89 & & & \\
\hline (p) & $0.0000)$ & & & \\
\hline
\end{tabular}


Gerenciamento de Resultados por Decisões Operacionais no Novo Mercado do Brasil: Uma Análise da Influência de Auditorias Big Four e não Big Four

Assim como a equação (4), apenas o MTB se mostra significante $(0,1 \%)$ e com sinal positivo, e o estimador para AUDIT com sinal negativo, porém não significante estatisticamente. Dessa forma a interpretação se dá de maneira similar para o nível anormal de produção, ao passo que não há diferença no nível dessa proxy quando a empresa é auditada por Big Four ou não Big Four. Sendo assim, a hipótese 3 é também refutada.

Os resultados apresentados para as três hipóteses revelam que o nível de gerenciamento de resultados por decisão operacional - seja por fluxo de caixa operacional, despesas de venda, gerais e administrativas ou produção - para empresas brasileiras do novo mercado não é afetado pela firma de auditoria pertencer ou não ao grupo das Big Four.

\section{CONCLUSÃO}

O tema gerenciamento de resultado tem sido bastante debatido na literatura contábil nos últimos anos. Entretanto nenhum trabalho no Brasil se propôs a verificar a influência das empresas de auditoria no nível de gerenciamento de resultados por decisões operacionais no período pós-IFRS.

Este trabalho teve como objetivo geral verificar se há diferença no nível de gerenciamento de resultados por decisões operacionais nas companhias abertas brasileiras do novo mercado quando auditadas por Big Four ou não Big Four. Para isso, foi elaborada uma pesquisa documental com as demonstrações financeiras das empresas-alvo para obtenção dos dados primários necessários. Com a amostra de dados coletadas dos relatórios dos anos de 2010 a 2017 e organizada por meio de dados em painel, resultando em 515 observações de empresasano, foi utilizado o método dos Mínimos Quadrados Generalizados para obtenção dos estimadores de cada variável e possibilitar as inferências sobre estes.

O estudo não encontrou evidências para diferença no nível de gerenciamento de resultados por decisões operacionais, seja através daquelas vinculadas ao Fluxo de Caixa Operacional, Despesas de Venda, Gerais e Administrativas ou Produção, quando da empresa ser auditada por uma firma de auditoria Big Four ou não Big Four. Apesar do coeficiente atrelado a variável AUDIT ser negativo para as regressões acerca do nível anormal de FCO e PROD, não podemos afirmar que as auditorias Big Four são inibidoras de gerenciamento de resultado por decisões operacionais, pois esse coeficiente é estatisticamente insignificante.

Devido a diferenças metodológicas na avaliação do problema de pesquisa aplicado ao período pós-IFRS no Brasil, não é possível comparar diretamente os resultados com outros trabalhos, apenas as pesquisas similares com intervalo temporal e metodologias diferentes. Nesse caso, há uma confirmação das conclusões, como apontado em Martinez (2011), o qual apenas o nível de gerenciamento por decisões contábeis reduz com a presença de firmas de autoria Big Four, sem impacto quando analisado o gerenciamento por decisões operacionais para o período de 1998 a 2004.

O trabalho se limita em relação ao estudo apenas de empresas listadas no Novo Mercado da B3, ou seja, aquelas que possuem um maior nível de governança corporativa. Esse fato pode interferir na possível influência que uma empresa de auditoria possa ter sobre os relatórios financeiros e consequentemente no gerenciamento de resultado.

Diante disso, recomenda-se para pesquisas futuras a análise de uma amostra ampliada, contemplando diferentes níveis de governança corporativa para identificar a influência da firma de auditoria para as empresas brasileiras de capital aberto em geral no tocante ao gerenciamento de resultados por decisões operacionais. Além disso, faz-se necessário também uma análise segmentada por setor econômico das empresas, uma vez que a regressão por setor torna-se um requisito indispensável para a qualidade das estimativas dada às especificidades de cada indústria (MARTINEZ; CARDOSO, 2009). 
Sena, Dias Filho e Moreira (2020)

Gerenciamento de Resultados por Decisões Operacionais no Novo Mercado do Brasil:

Uma Análise da Influência de Auditorias Big Four e não Big Four

\section{REFERÊNCIAS}

ALMEIDA, J. E. F. de; ALMEIDA, J. C. G de. Auditoria e earnings management: estudo empírico nas empresas abertas auditadas pelas big four e demais firmas de auditoria. Revista Contabilidade \& Finanças, v. 20, n. 50, p. 62-74, 2009.

ANDERSON, M. C.; BANKER, R. D.; JANAKIRAMAN, S. N. Are selling, general, and administrative costs "sticky"? Journal of Accounting Research, v. 41, n. 1, p. 47-63, 2003.

BARALDI, A.; PEREIRA, A. da R. Auditoria como alternativa de identificação de gerenciamento de resultados na Medic Assistent-rs. 2011. 19 f. Trabalho de Conclusão de Curso (Graduação em Ciências Contábeis) - Universidade de Cruz Alta, Rio Grande do Sul.

BRAUNBECK, G. O. Determinantes da qualidade das auditorias independentes no Brasil. 2010. 129 f. Tese (Doutorado em contabilidade e atuária) - Faculdade de Economia, Administração e Contabilidade. Universidade de São Paulo, São Paulo.

BRAUNBECK, G. O.; CARVALHO, L. N. Auditoria Independente. In: LOPES, A. B.; IUDÍCIBUS, S. Teoria Avançada da Contabilidade. São Paulo: Atlas, 2012. cap. 8, p. 303329.

COHEN, D. A.; DEY, A.; LYS, T. Z. Real and accrual-based earnings management in the pre-and post-Sarbanes-Oxley periods. The accounting review, v. 83, n. 3, p. 757-787, 2008.

CUPERTINO, C. M. Gerenciamento de resultados por decisões operacionais no mercado brasileiro de capitais. 2013. 126 f. Tese (doutorado) - Programa de Pós-Graduação em Administração. Universidade Federal de Santa Catarina, Florianópolis.

DEANGELO, L. E. Auditor size and audit quality. Journal of accounting and economics, v. 3, n. 3, p. 183-199, 1981.

DECHOW, P. M.; KOTHARI, S. P.; WATTS, R. L. The relation between earnings and cash flows. Journal of accounting and Economics, v. 25, n. 2, p. 133-168, 1998.

DECHOW, P.; GE, W.; SCHRAND, C. Understanding earnings quality: A review of the proxies, their determinants and their consequences. Journal of accounting and economics, v. 50, n. 2-3, p. 344-401, 2010.

DECHOW, P. M.; SLOAN, R. G.; SWEENEY, A. P. Causes and consequences of earnings manipulation: An analysis of firms subject to enforcement actions by the SEC. Contemporary accounting research, v. 13, n. 1, p. 1-36, 1996.

Ge, W. Essays on real earnings management. 2009. 124 f. Tese (Doutorado) - McGill University. Desautels Faculty of Management, Quebec.

GRAHAM, J. R.; HARVEY, C. R.; RAJGOPAL, S. The economic implications of corporate financial reporting. Journal of accounting and economics, v. 40, n. 1-3, p. 3-73, 2005.

GOULART, A. M. C. Gerenciamento de resultados contábeis em instituições financeiras no Brasil. 2007. 219 f. Tese (Doutorado em contabilidade e atuária) - Faculdade de Economia, Administração e Contabilidade. Universidade de São Paulo, São Paulo.

GUNNY, K. A. The relation between earnings management using real activities manipulation and future performance: Evidence from meeting earnings benchmarks. Contemporary Accounting Research, v. 27, n. 3, p. 855-888, 2010.

HEALY, P. M. The effect of bonus schemes on accounting decisions. Journal of accounting and economics, v. 7, n. 1-3, p. 85-107, 1985. 
Sena, Dias Filho e Moreira (2020)

Gerenciamento de Resultados por Decisões Operacionais no Novo Mercado do Brasil: Uma Análise da Influência de Auditorias Big Four e não Big Four

HEALY, P. M.; PALEPU, K. G. Information asymmetry, corporate disclosure, and the capital markets: A review of the empirical disclosure literature. Journal of accounting and economics, v. 31, n. 1-3, p. 405-440, 2001.

JONES, J. J. Earnings management during import relief investigations. Journal of accounting research, v. 29, n. 2, p. 193-228, 1991.

JENSEN, M. C.; MECKLING, W. H. Theory of the firm: Managerial behavior, agency costs and ownership structure. Journal of financial economics, v. 3, n. 4, p. 305-360, 1976.

MACHADO, Nathan Vasconcellos de Almeida Rezende. Análise da sensibilidade do Earnings Response Coefficient (ERC) à adoção das IFRS no mercado brasileiro. 2018. 173f. Dissertação (Mestrado em Controladoria e Contabilidade). Universidade Federal de Minas Gerais, Belo Horizonte, MG, 2018.

MARTINEZ, A. L. Gerenciamento dos resultados contábeis: estudo empírico das companhias abertas brasileiras. 2001. $153 \mathrm{f}$. Tese (doutorado) Tese (Doutorado em contabilidade e atuária) - Faculdade de Economia, Administração e Contabilidade. Universidade de São Paulo, São Paulo.

Do corporate governance special listing segments and auditing curb real and accrualbased earnings management? Evidence from Brazil. Revista Universo Contábil, v. 7, n. 4, p. 98-117, 2011.

Gerenciamento de resultados no Brasil: um survey da literatura. BBR-Brazilian Business Review, v. 10, n. 4, p. 1-31, 2013.

MARTINEZ, A. L.; CARDOSO, R. L. Gerenciamento da informação contábil no Brasil mediante decisões operacionais. REAd-Revista Eletrônica de Administração, v. 15, n. 3, p. 601-626, 2009.

MATSUMOTO, A. S.; PARREIRA, E. M. Uma pesquisa sobre o Gerenciamento de Resultados Contábeis: causas e conseqüências. Revista Contabilidade, Gestão e Governança, v. 10, n. 1, p. 141-157, 2009.

PELEIAS, Fabiola D.'Agostini. Mecanismos linguísticos (des) favoráveis para a readability das demonstrações financeiras: uma análise das empresas listadas no mercado de capitais brasileiro. 2017. 107f. Dissertação (Mestrado em Ciências Contábeis). Fundação Escola de Comércio Álvares Penteado, São Paulo, SP, 2017.

QUEIROZ, M. M. M. D. Gerenciamento de resultados: uma análise setorial do nível nas empresas auditadas por big four e não big four listadas na BM\&FBOVESPA. 2016. 42 f. Trabalho de Conclusão de Curso (Graduação em Ciências Contábeis) - Departamento de Ciências Contábeis. Universidade Federal do Rio Grande do Norte, Natal.

RAMOS, G. M.; MARTINEZ, A. L. Governança corporativa. Revista Contemporânea de Contabilidade, v. 3, n. 6, p. 143-164, 2006.

REIS, E. M. DOS; CUNHA, J. V. A. DA; RIBEIRO, D. M. Análise do gerenciamento de resultados por meio de decisões operacionais nas empresas componentes do IBrX-Índice Brasil. Advances in Scientific and Applied Accounting, v. 7, n. 2, p. 201-223, 2014.

REIS, E. M. DOS; LAMOUNIER, W. M.; BRESSAN, V. G. F. Evitar Divulgar Perdas: Um Estudo Empírico do Gerenciamento de Resultados por Meio de Decisões

Operacionais. Revista Contabilidade \& Finanças, v. 26, n. 69, p. 247-260, 2015. 
ROYCHOWDHURY, S. Earnings management through real activities manipulation. Journal of accounting and economics, v. 42, n. 3, p. 335-370, 2006.

SANTANA, A. G. et al. Auditoria independente e a qualidade da informação na divulgação das demonstrações contábeis: estudo comparativo entre empresas brasileiras auditadas pelas Big Four e Não Big Four. Revista de Contabilidade do Mestrado em Ciências Contábeis da UERJ, v. 19, n. 3, p. 70-87, 2016.

SANTOS, P. S.; VERHAGEM, J. A.; BEZERRA, F. A. Gerenciamento de resultados por meio de decisões operacionais e a governança corporativa: análise das indústrias siderúrgicas e metalúrgicas brasileiras. Revista de Contabilidade e Organizações, v. 5, n. 13, p. 55-74, 2011.

SCHIPPER, K. Earnings management. Accounting horizons, n. 3, v. 4, p. 91-102, 1989.

SILVA, A. D. et al. Influência da auditoria sobre o gerenciamento de resultados. Revista de

Contabilidade do Mestrado em Ciências Contábeis da UERJ, v. 19, n. 3, p. 59-69, 2016.

SILVA, V. C. D. A adoção das IFRS impacta no gerenciamento de resultados?

Evidências do mercado de capitais brasileiro. 2015. 86 f. Dissertação (Mestrado em Ciências Contábeis) - Programa de Pós Graduação em Ciências Contábeis. Universidade Federal do Espírito Santo, Vitória.

WATTS, R. L.; ZIMMERMAN, J. L. Agency problems, auditing, and the theory of the firm: Some evidence. The Journal of Law and Economics, v. 26, n. 3, p. 613-633, 1983.

Positive accounting theory. New Jersey: Prentice-Hall, 1986.

Positive accounting theory: a ten year perspective. The Accounting Review, v. 65, p. 131-156, 1990.

XU, Z. Three essays on real earnings management. 2007. Tese (Doutorado) - Culverhouse School of Accountancy. The University of Alabama, Tuscaloosa.

ZANG, A. Y. Evidence on the trade-off between real activities manipulation and accrualbased earnings management. The Accounting Review, v. 87, n. 2, p. 675-703, 2011. 